\title{
Minimally invasive versus open distal pancreatectomy for pancreatic neuroendocrine tumors: An analysis from the U.S. neuroendocrine tumor study group
}

\author{
Xu-Feng Zhang MD PhD ${ }^{1,2}$ ( ) Alexandra G. Lopez-Aguiar $\mathrm{MD}^{3}$ | George Poultsides $\mathrm{MD}^{4} \mid$ \\ Eleftherios Makris MD ${ }^{4}$ Flavio Rocha MD ${ }^{5}$ | Zaheer Kanji MD | Sharon Weber MD ${ }^{6}$ |

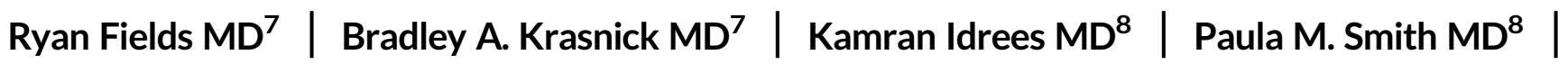 \\ Cliff Cho MD ${ }^{9}$ | Carl R. Schmidt MD $^{2}$ | Shishir K. Maithel MD ${ }^{3}$ | \\ Timothy M. Pawlik MD MPH PhD² | The United States Neuroendocrine Tumor Study Group \\ ${ }^{1}$ Department of Hepatobiliary Surgery and Institute of Advanced Surgical Technology and Engineering, The First Affiliated Hospital of Xi'an Jiaotong University, \\ Xi'an, China \\ ${ }^{2}$ Department of Surgery, The Ohio State University Wexner Medical Center and James Comprehensive Cancer Center, Columbus, Ohio \\ ${ }^{3}$ Division of Surgical Oncology, Department of Surgery, Winship Cancer Institute, Emory University, Atlanta, Georgia \\ ${ }^{4}$ Department of Surgery, Stanford University, Palo Alto, California \\ ${ }^{5}$ Department of Surgery, Virginia Mason Medical Center, Seattle, Washington \\ ${ }^{6}$ Department of Surgery, University of Wisconsin School of Medicine and Public Health, Madison, Wisconsin \\ ${ }^{7}$ Department of Surgery, Washington University School of Medicine, St. Louis, Wisconsin \\ ${ }^{8}$ Division of Surgical Oncology, Department of Surgery, Vanderbilt University, Nashville, Tennessee \\ ${ }^{9}$ Division of Hepatopancreatobiliary and Advanced Gastrointestinal Surgery, Department of Surgery, University of Michigan, Ann Arbor, Michigan
}

\section{Correspondence}

Timothy M. Pawlik, MD, MPH, PhD, FACS,

FRACS (Hon.), Professor and Chair,

Department of Surgery, The Urban Meyer III and Shelley Meyer Chair for Cancer Research, Professor of Surgery, Oncology, Health Services Management and Policy, The Ohio State University, Wexner Medical Center, 395 W. 12th Ave., Suite 670, Columbus 43210, Ohio.

Email: tim.pawlik@osumc.edu

Funding information

Clinical Research Award of the First Affiliated Hospital of Xi'an Jiaotong University of China, Grant/Award Number: XJTU1AF-CRF2017-004

\begin{abstract}
Background: To determine short- and long-term oncologic outcomes after minimally invasive distal pancreatectomy (MIDP) with open distal pancreatectomy (ODP) for the treatment of pancreatic neuroendocrine tumor ( $p N E T$ ).

Methods: The data of the patients who underwent curative MIDP or ODP for pNET between 2000 and 2016 were collected from a multi-institutional database. Propensity score matching (PSM) was used to generate 1:1 matched patients with MIDP and ODP.

Results: A total of 576 patients undergoing curative DP for pNET were included. Two hundred and fourteen (37.2\%) patients underwent MIDP, whereas 362 (62.8\%) underwent ODP. MIDP was increasingly performed over time (20002004: 9.3\% vs 2013-2016: 54.8\%; $P<0.01)$. In the matched cohort $(n=141$ in each group), patients who underwent MIDP had less blood loss (median, 100 vs $200 \mathrm{~mL}, P<0.001)$, lower incidence of Clavien-Dindo $\geq$ III complications (12.1\% vs $24.8 \%, P=0.026$ ), and a shorter hospital stay versus ODP (median, 4 versus 7 days, $P=0.026)$. Patients who underwent MIDP had a lower incidence of recurrence (5-year cumulative recurrence, $10.1 \%$ vs $31.1 \%, P<0.001$ ), yet equivalent overall survival (OS) rate (5-year OS, $92.1 \%$ vs $90.9 \%, P=0.550)$ compared with patients who underwent OPD.
\end{abstract}


Conclusion: Patients undergoing MIDP over ODP in the treatment of pNET had comparable oncologic surgical metrics, as well as similar long-term OS.

KEYWORDS

laparoscopic, neuroendocrine tumor, pancreas, prognosis, robotic

\section{1 | INTRODUCTION}

Pancreatic neuroendocrine tumors (pNET) account for $2 \%$ to $4 \%$ of all pancreatic neoplasms diagnosed in the general population. ${ }^{1,2}$ Over the last two decades, the incidence of pNET has increased significantly because of the widespread use of cross-sectional imaging. ${ }^{3}$ In fact, the incidence of pNET currently is about 0.48 per 100,000 persons and pNET is the leading cause of cancerrelated deaths in the United States. ${ }^{4,5}$ Although most cases are sporadic, $10 \%-30 \%$ are associated with genetic syndromes, such as multiple endocrine neoplasia 1 syndrome, and Von Hippel-Lindau disease. ${ }^{1,2}$

Surgical resection remains the optimal curative modality for pNET and is the treatment of choice even among certain patients with locally advanced or metastatic disease. ${ }^{6-9}$ The surgical approach can consist of "typical" and "atypical" resections depending on the number, size, and location of the tumor(s). Typical resection includes pancreaticoduodenectomy (PD) for tumors located in the pancreatic head, distal pancreatectomy (DP) for tumors located in the body and tail, and rarely total pancreatectomy (TP) when tumors spread within the whole gland. ${ }^{3,10}$ Atypical resection includes enucleation and central pancreatectomy $(\mathrm{CP})$ and is more often utilized when tumors are small $(<2-3 \mathrm{~cm})$, benign, well-circumscribed, as well as not adjacent to the duct of Wirsung so that the duct can remain intact after tumor resection. ${ }^{10,11}$

Because the first laparoscopic pancreatic surgery performed by Cuschieri in $1994,{ }^{12}$ laparoscopic surgery has been increasingly utilized in pancreatic surgery because of its minimal invasiveness versus open procedures. ${ }^{3,13-16}$ Because of its overall relatively low incidence, the assessment of short- and long-term outcomes after laparoscopic versus open surgery for pNET have been limited. Especially, most data have been derived from small retrospective studies that have yielded disparate results..$^{3,10,15,16}$ In addition, the heterogeneity of patients who had different tumor locations requiring different surgical procedures may induce selection bias when comparing minimally invasive versus open surgical approaches. In addition, most previous studies largely focused on short-term outcomes with the long-term oncological outcomes of minimally invasive vs open surgery for pNETs remaining largely undetermined. ${ }^{10}$ Therefore, the objective of the current study was to define short- and long-term outcomes after minimally invasive distal pancreatectomy (MIDP) vs open distal pancreatectomy (ODP) among patients with pNETs located in the body and tail of the pancreas.

\section{PATIENTS AND METHODS}

\section{1 | Design and patients}

Patients who underwent curative resection for pNET between 2000 and 2016 were identified from the U.S. Neuroendocrine Tumor Study Group (US-NETSG). The US-NETSG included The Ohio State University Wexner Medical Center and James Comprehensive Cancer Center, Columbus, OH; Winship Cancer Institute, Emory University, Atlanta, GA; Stanford University, Palo Alto, CA; Virginia Mason Medical Center, Seattle, WA; University of Wisconsin, School of Medicine and Public Health, Madison, WI; Washington University, School of Medicine, St. Louis, MO; Vanderbilt University, Nashville, $\mathrm{TN}$; University of Michigan, Ann Arbor, MI. ${ }^{17}$ The Institutional Review Board of each participating institution approved the study.

All patients were pathologically diagnosed with pNET on the basis of conventional histology and immunohistochemical findings (chromogranin A, synaptophysin, and Ki-67). Standard patient demographic, clinicopathologic, and perioperative data were collected on the basis of a prospectively maintained database.

\subsection{Surgical treatment and postoperative surveillance}

All surgeries were performed by specialized physicians. Choice of laparoscopic/robotic or open surgery was mostly determined by tumor factors, as well as surgeon preference. Operative time was defined as the time duration between the first incision and skin closure. Margin status was determined by the pathologist on the basis of the examination of all specimen margin sites on permanent sections. An RO resection was defined as a minimum margin length of $>1 \mathrm{~mm}$; the microscopic presence of the tumor at the margin or a minimum margin length of $\leq 1 \mathrm{~mm}$ was designated as an $\mathrm{R} 1$ resection. The inability to resect all gross residual disease was defined as an R2 resection. ${ }^{18}$ Tumor-related characteristics, including maximal tumor diameter, number, location, tumor morphology, histological grade, lymph-vascular/perineural invasion, Ki-67, mitotic rate, nodal status were recorded on the basis of final pathology. All cases were reviewed and classified according to the World Health Organization (WHO) criteria. ${ }^{19}$

Postoperative morbidity was graded according to the ClavienDindo classification. ${ }^{20}$ The definition of postoperative hemorrhage and the pancreatic fistula was on the basis of the International Study Group of Pancreatic Surgery. ${ }^{21,22}$ All patients were followed regularly in each participating institution. Disease recurrence was 
defined as identification of suspicious imaging findings on postoperative surveillance or biopsy-proven recurrent pNET. Overall survival (OS) was calculated from the date of surgery to the date of death or date of last follow-up.

\section{3 | Statistical analysis}

Numerical variables were expressed as medians with interquartile ranges (IQR) and compared with Student $t$ test or the Mann-Whitney $U$ test between the two groups. Nominal variables were expressed as number and percentages and compared with the Chi-squared test or Fisher's exact test. Survival probabilities were estimated by KaplanMeier methodology and compared by log-rank analysis. Two-tailed $P$-values $<0.05$ were considered statistically significant. Potential risk factors associated with OS and tumor recurrence were identified using univariate and multivariable Cox hazard regression models. Results were reported as hazard ratios (HR) and 95\% confidence intervals $(95 \% \mathrm{Cl})$. Propensity score matching (PSM) was used to mitigate selection bias. Specifically, variables potentially affecting long-term outcomes were utilized in the propensity score on the basis of identification in the logistic regression analysis. The propensity score analysis with 1:1 matching was performed without replacement using a caliper with a width 0.05 of the standard deviation to generate matched pairs of the patients. In all analyses, two-tailed $P$ value $<0.05$ was considered statistically significant. Statistical analysis was carried out using SPSS 22.0 (Chicago, IL).

\section{RESULTS}

A total of 1020 patients undergoing curative-intent resection for pNET were included (Figure 1). Classic PD was performed in 129 patients, pylorus-preserving pancreaticoduodenectomy (PPPD) in 159 patients, CP in 32 patients, DP in 576 patients, TP in 17 patients, and tumor enucleation in 107 patients. Minimally invasive surgery (MIS) was mainly performed among patients undergoing DP; 214 patients who underwent MIDP, 25 patients who underwent laparoscopic/robotic converted to ODP, and 337 patients who underwent ODP were included in the analytic cohort. Utilization of MIDP increased over time (2000-2004, 9.3\%; 2005-2008, 14.5\%; 2009-2012, 41.8\%; 2013-2016, 54.8\%, $P<0.01$ for trend; Figure 2).

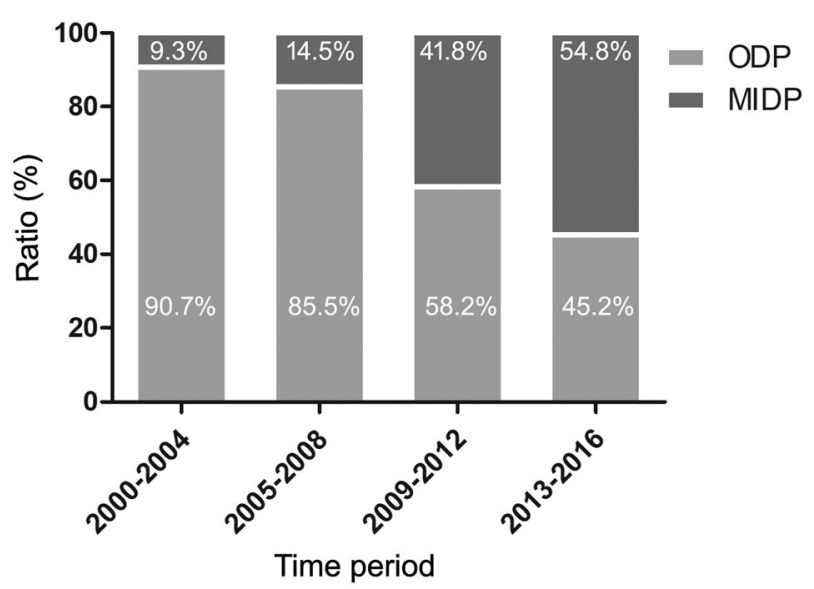

patient number

$\begin{array}{llll}43 & 131 & 225 & 177\end{array}$

FIGURE 2 Utilization of minimally invasive distal pancreatectomy (MIDP) and open distal pancreatectomy (ODP) for pancreatic neuroendocrine tumor ( $\mathrm{pNET}$ ) at different time periods

In addition, the conversion rate of MIDP to open decreased (20002004, 20.0\%; 2005-2008, 29.6\%, 2009-2012, 7.8\%, 2013-2016, 7.6\%; Table S1).

\section{1 | Analytic cohort}

Among the 576 patients who underwent curative DP for pNET, median age was 58 (IQR 48-66) years with a slight female predominance $(n=314,54.5 \%)$. A majority of patients $(n=490$, $85.1 \%)$ presented with nonfunctional tumors and had no specific genetic syndrome ( $n=512,88.9 \%)$; almost half of patients ( $n=276,47.9 \%$ ) were symptomatic. Most patients had a single ( $n=510,88.5 \%$ ), small $(\leq 3 \mathrm{~cm}, \mathrm{n}=375,65.1 \%)$ mass and a welldifferentiated tumor $(n=443,76.9 \%)$. Most tumors $(n=386$, $67.0 \%)$ were located in the pancreatic tail. After the surgery, roughly one-half of patients ( $n=304,52.8 \%$ ) experienced at least one complication, whereas $113(19.6 \%)$ patients experienced a severe complication (Clavien-Dindo III-V); 22 (3.8\%) patients required reoperation because of bleeding $(n=9)$, intra-abdominal abscess $(n=6)$, intestinal obstruction $(n=3)$, and unknown reasons ( $n=4$; Table 1).
FIGURE 1 Study scenario and patient selection. CP, central pancreatectomy; DP, distal pancreatectomy; MIS, minimally invasive surgery; PD, pancreaticoduodenectomy; PNET, pancreatic neuroendocrine tumor; PPPD, pylorus-preserving pancreaticoduodenectomy; TP, total pancreatectomy

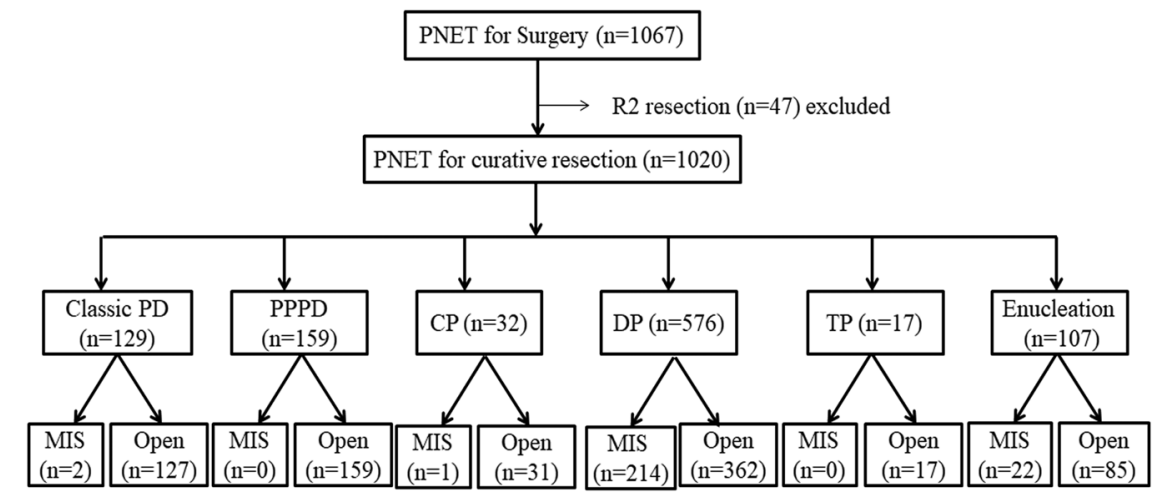


TABLE 1 Clinicopathologic characteristics and operation details of patients undergoing minimally invasive distal pancreatectomy (MIDP) versus open distal pancreatectomy (ODP) for pancreatic neuroendocrine tumors (pNETs)

\begin{tabular}{|c|c|c|c|c|}
\hline & $\begin{array}{l}\text { Overall } \\
(n=576)\end{array}$ & MIDP $(n=214)$ & ODP $(n=362)$ & $P$ value \\
\hline Age, y & $58(48-66)$ & $59(50-66)$ & $56(47-65)$ & 0.414 \\
\hline Male & 262 (45.5\%) & 93 (43.5\%) & $169(46.7 \%)$ & \\
\hline Female & $314(54.5 \%)$ & $121(56.5 \%)$ & 193 (53.3\%) & \\
\hline Nonfunctional & 490 (85.1\%) & 179 (83.6\%) & 311 (85.9\%) & \\
\hline Functional & $73(12.7 \%)$ & 32 (15.0\%) & 41 (11.3\%) & \\
\hline NA & $13(2.3 \%)$ & $3(1.4 \%)$ & $10(2.8 \%)$ & \\
\hline Genetic syndrome & & & & 0.064 \\
\hline NA & $12(2.1 \%)$ & $3(1.4 \%)$ & 9 (2.5\%) & \\
\hline Symptomatic & $276(47.9 \%)$ & 99 (46.3\%) & 177 (48.9\%) & 0.486 \\
\hline Primary location & & & & $<0.001$ \\
\hline Neck/body & $162(28.1 \%)$ & $46(21.5 \%)$ & $116(32.0 \%)$ & \\
\hline Tail & $386(67.0 \%)$ & 165 (77.1\%) & $221(61.0 \%)$ & \\
\hline Multiple location & $28(4.9 \%)$ & $3(1.4 \%)$ & $25(6.9 \%)$ & \\
\hline Largest tumor size, $\mathrm{cm}$ & & & & 0.001 \\
\hline$\leq 3$ & $375(65.1 \%)$ & $157(73.4 \%)$ & $218(60.2 \%)$ & \\
\hline Major vascular resection & 11 (1.9\%) & 0 & $11(3.0 \%)$ & 0.009 \\
\hline Pancreatic transection & & & & $<0.001$ \\
\hline Hand-sewn & $30(5.2 \%)$ & $3(1.4 \%)$ & $27(7.5 \%)$ & \\
\hline Stapled with no suture & $220(38.2 \%)$ & $113(52.8 \%)$ & 107 (29.6\%) & \\
\hline Stapled with reinforcement & $160(27.8 \%)$ & 49 (22.9\%) & $111(30.6 \%)$ & \\
\hline Other procedures & $23(4.0 \%)$ & 7 (3.3\%) & $16(4.4 \%)$ & \\
\hline Missing & $143(24.8 \%)$ & $42(19.6 \%)$ & 101 (27.9\%) & \\
\hline Intraoperative abdominal drainage & $518(89.9 \%)$ & $189(88.3 \%)$ & $329(90.9 \%)$ & 0.132 \\
\hline Operation time, $\min$ & $210(179-263)$ & $210(180-258)$ & $210(177-266)$ & 0.652 \\
\hline Estimated blood loss, $\mathrm{mL}$ & $200(100-400)$ & $100(50-150)$ & $300(150-500)$ & $<0.001$ \\
\hline Lymphadenectomy & 509 (88.4\%) & $187(87.4 \%)$ & $322(89.0 \%)$ & 0.331 \\
\hline No. of lymph node retrieved & $9(4-14)$ & $9(4-14)$ & $8(4-14)$ & 0.709 \\
\hline Lymph nodes status & & & & 0.001 \\
\hline Negative & 411 (71.4\%) & $166(77.6 \%)$ & $245(67.7 \%)$ & \\
\hline Positive & 99 (17.2\%) & $22(10.3 \%)$ & 77 (21.3\%) & \\
\hline Tumor differentiation & & & & 0.824 \\
\hline Well differentiated & $443(76.9 \%)$ & $173(80.8 \%)$ & $270(74.6 \%)$ & \\
\hline
\end{tabular}


TABLE 1 (Continued)

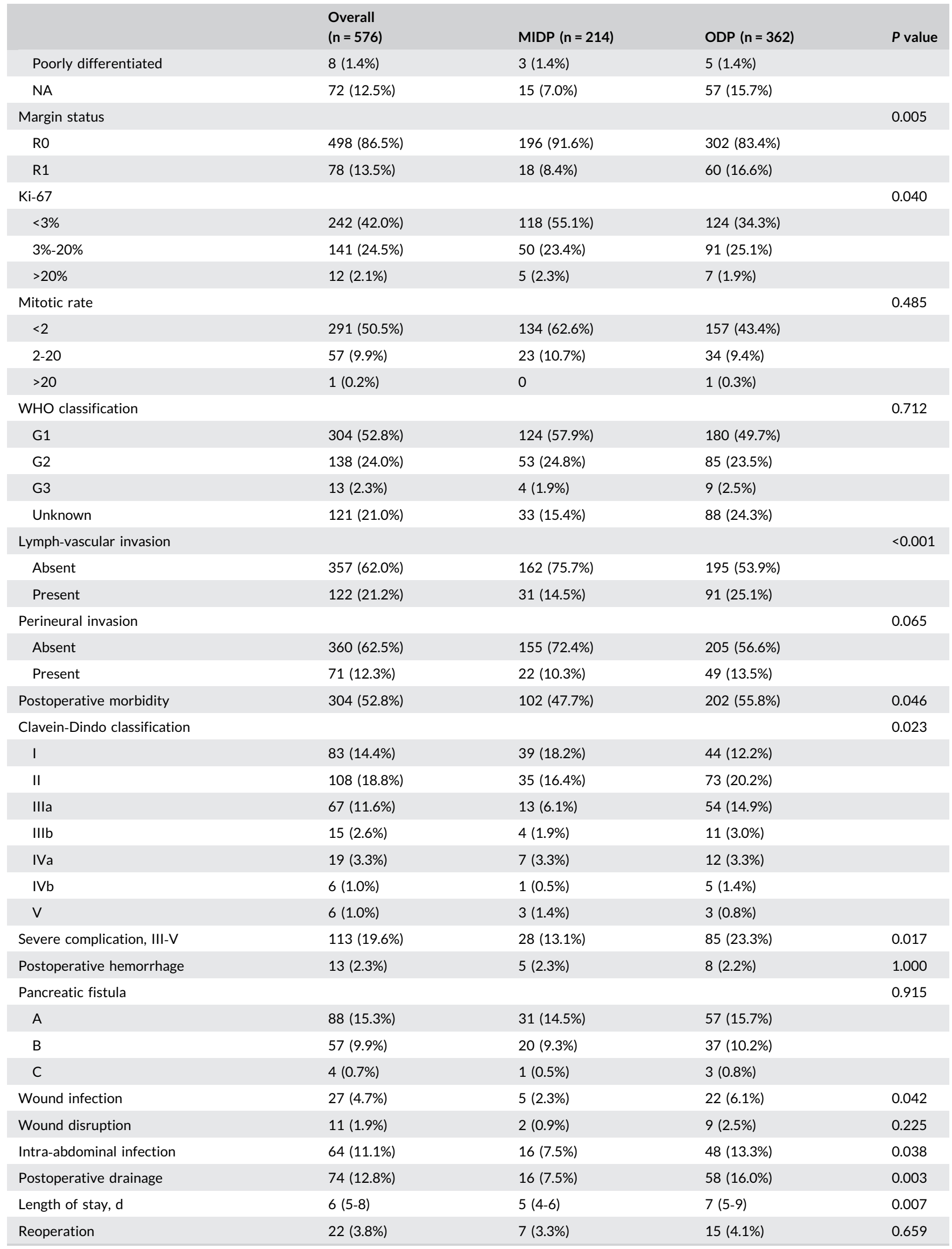

Abbreviations: MEN, multiple endocrine neoplasia; MIDP, minimally invasive distal pancreatectomy; ODP, open distal pancreatectomy; VHL, Von Hippel-Lindau. 
The clinicopathologic characteristics and surgical details among patients undergoing MIDP $(n=214,37.2 \%)$ and ODP $(n=362,62.8 \%)$ were assessed (Table 1). Compared with OPD, MIDP was more likely to be performed among patients with a single (MIDP $93.9 \%$ vs OPD $85.4 \%, P=0.002$ ) smaller tumor ( $\leq 3 \mathrm{~cm}$, MIDP $73.4 \%$ vs OPD $60.2 \%$, $P=0.001$ ). In addition, concomitant splenectomy (MIDP $75.7 \%$ vs OPD $87.0 \%, P=0.001$ ) and additional pancreatic enucleation (MIDP $0.5 \%$ vs OPD $4.1 \%, P=0.002$ ) were less frequently performed among patients undergoing MIDP vs OPD. MIDP was associated with less intraoperative blood loss than OPD (median, 100 versus $300 \mathrm{~mL}$, $P<0.001$ ), yet operative time (median, 210 minutes versus 210 ), as well as final $\mathrm{WHO}$ classification and tumor grade were similar among patients undergoing MIDP vs OPD (all $P>0.05$ ). The number of lymph node retrieved (median, 9 vs $8, P=0.709$ ) were equivalent among MIDP and OPD. Interestingly, the median number of lymph nodes harvested increased over time among patients undergoing both MIDP (2000-2004, 3 nodes vs 2013-2016, 10 nodes) and OPD (2000-2004, 3 nodes vs 2013-2016, 13 nodes; Table S1, both $P<0.05)$. In contrast, the incidence of $R 0$ resection was higher among patients undergoing MIDP vs OPD (91.6\% vs $83.4 \%, P=0.005)$.

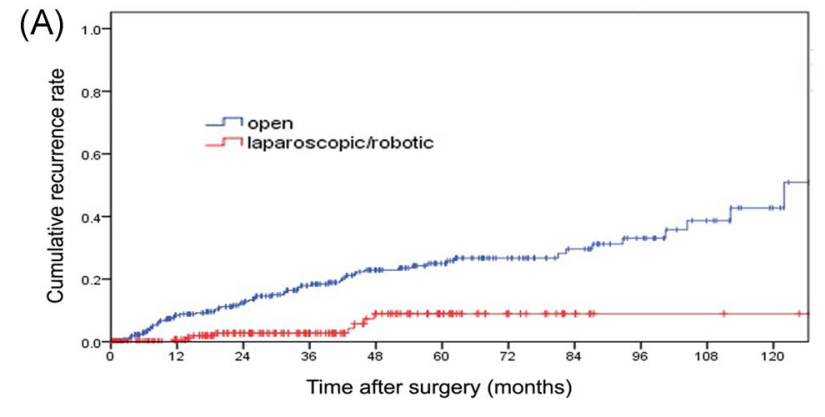
Patients at risk

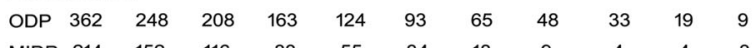
$\begin{array}{lllllllllll}\text { MIDP } 214 & 152 & 116 & 83 & 55 & 34 & 18 & 9 & 4 & 4 & 3\end{array}$
MIDP was also associated with lower overall postoperative morbidity (47.7\% vs $55.8 \%, P=0.046$ ), as well as a lower incidence of wound infection ( $2.3 \%$ vs $6.1 \%, P=0.042$ ), severe complications $(13.1 \%$ vs 23.3\%, $P=0.017$ ), and a shorter in-hospital stay (median 5 days vs 7 days, $P=0.007$ ) vs OPD (Table 1 ). Of note, while the length-of-stay did not differ over time in the OPD group, the length-of-stay did decrease from a median of 9 days to 4 days in the MIDP group (Table S1).

\section{2 | Long-term outcomes}

With a median follow-up of 35.4 months (IQR 11.9-62.0 months), 77 (13.4\%) patients developed recurrence (MIDP, 3.7\% vs ODP, 19.1\%) and 53 (9.2\%) patients died (MIDP, 5.1\% vs ODP, 11.6\%). Overall 3-, 5 -, and 10-year cumulative recurrence was $2.7 \%, 8.9 \%$, and $8.9 \%$ among patients who had MIDP vs $18.4 \%, 25.9 \%$, and $42.7 \%$ among individuals who underwent ODP, respectively $(\mathrm{HR} 0.2,95 \% \mathrm{Cl}$, 0.1-0.5, $P<0.001$; Figure 3A). In contrast, OS was comparable among patients undergoing MIDP and ODP (3-, 5-, and 10-year OS, MIDP

\section{After propensity score matching}
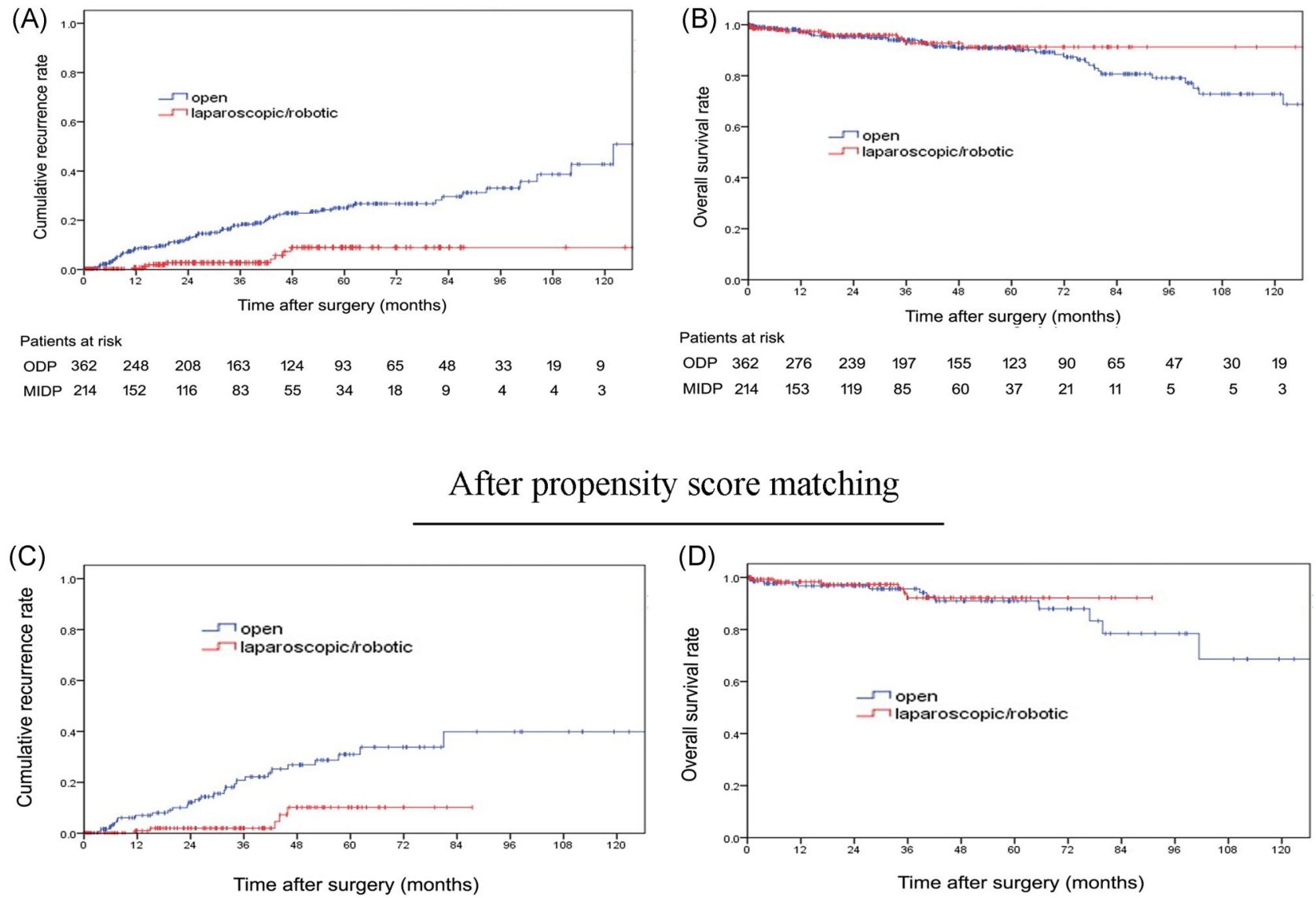

Patients at risk

$\begin{array}{llllllllllll}\text { ODP } & 141 & 98 & 82 & 58 & 44 & 25 & 18 & 10 & 9 & 6 & 2\end{array}$

Patients at risk

$\begin{array}{ccccccccccccccccccccccccc}\text { MIDP } & 141 & 98 & 76 & 49 & 29 & 15 & 4 & 1 & - & - & - & \text { MIDP } 141 & 99 & 78 & 50 & 32 & 17 & 6 & 2 & - & - & -\end{array}$

FIGURE 3 Cumulative tumor recurrence rate (A) and overall survival (B) among patients undergoing minimally invasive distal pancreatectomy (MIDP) and open distal pancreatectomy (ODP) for pancreatic neuroendocrine tumor (pNET) in an unadjusted cohort. Cumulative tumor recurrence rate $(C)$ and overall survival $(D)$ among patients undergoing MIDP and ODP for pNET in adjusted cohort [Color figure can be viewed at wileyonlinelibrary.com] 
$92.8 \%, 91.2 \%$ and $91.2 \%$ vs ODP $94.0 \%, 90.1 \%$, and $72.9 \%, P=0.300$; Figure 3B).

As the baseline characteristics among patients undergoing MIDP and ODP were different, PSM was utilized to generate 141 pairs of matched patients with similar functional status, tumor size, number, classification, as well as proportion of splenectomy, vascular resection, number of lymph node evaluated, number of metastatic nodes, margin status, and lymph-vascular invasion (Table S2). In the propensity model, patients who underwent MIDP had less blood loss (median, MIDP 100 [105-500] vs ODP 200 [105-500] $\mathrm{mL}, P<0.001$ ) and a lower incidence of severe complications (Clavien-Dindo III-V; MIDP $12.1 \%$ vs ODP 24.8\%, $P=0.026$ ), although overall morbidity was equivalent (Table 2). Perhaps not surprisingly, MIDP remained associated with a shorter in-hospital stay vs ODP (median, $4^{4-6}$ vs $7^{5-9}$ days, $P=0.026$; Table 2). Of note, in the propensity model, patients who underwent MIDP had a lower incidence of recurrence (5-year cumulative recurrence rate, MIDP $10.1 \%$ vs ODP $31.1 \%, P<0.001$; Figure $3 C$ ), yet comparable OS (5-year OS, MIDP $92.1 \%$ vs ODP $90.9 \% P=0.550$; Figure 3D). In assessing the entire cohort on the multivariable analysis, history of a genetic syndrome (HR 2.5, $95 \% \mathrm{Cl}, 1.1-5.8, P=0.034)$ and tumor size $>3 \mathrm{~cm}(\mathrm{HR} 3.3,95 \%$ $\mathrm{Cl}, 1.4-7.4, P=0.005)$ were associated with increased risk of tumor recurrence, whereas MIDP (vs ODP, HR 0.3, 95\% Cl, 0.1-0.9, $P=0.033)$ was associated with decreased tumor recurrence (Table 3). While MIDOP vs ODP was not associated with OS, tumor characteristics, such as WHO G3 classification (ref. G1, HR 4.0, 95\% Cl, 1.2-13.2, $P=0.001$ ) and poor tumor differentiation (ref. well differentiation, $\mathrm{HR} 2.3,95 \% \mathrm{Cl}, 1.2-6.7, P=0.025$ ) were associated with worse OS (Table S3).

\section{4 | DISCUSSION}

While MIS has been increasingly adopted for the treatment of pancreatic disease, the benefits of MIDP among patients with pNET remain not well described. The current study was important because, using a large multi-institutional cohort, we noted that utilization of MIDP vs ODP in the treatment of pNET had dramatically increased over the last two decades in specialized centers throughout the United States. Perhaps more importantly, the data from the current study demonstrated the short-term clinical advantages of MIDP vs ODP for pNET, including decreased intraoperative blood loss, as well as less risk of overall and severe postoperative morbidity and a shorter length of stay. Of note, ODP was more frequently performed among patients with advanced disease in terms of tumor size, number, vascular resection, and nodal involvement. As such, patients who underwent ODP had a higher incidence of tumor recurrence than patients who underwent MIDP (5-year cumulative recurrence rate, ODP $31.1 \%$ vs MIDP $10.1 \%, P<0.001)$. However, on both PSM and multivariate analysis, after controlling for some of these disparate risk factors, OS was equivalent among patients who underwent MIDP vs ODP.
TABLE 2 Postoperative morbidity of patients undergoing minimally invasive distal pancreatectomy (MIDP) versus open distal pancreatectomy (ODP) for pancreatic neuroendocrine tumors (pNETs) in the propensity model

\begin{tabular}{|c|c|c|c|}
\hline & $\begin{array}{l}\text { MIS } \\
(n=141)\end{array}$ & $\begin{array}{l}\text { Open } \\
(n=141)\end{array}$ & $P$ value \\
\hline Postoperative morbidity & $68(48.2 \%)$ & 82 (58.2\%) & 0.094 \\
\hline $\begin{array}{c}\text { Clavein-Dindo } \\
\text { classification }\end{array}$ & & & 0.072 \\
\hline 1 & $30(21.3 \%)$ & 17 (12.1\%) & \\
\hline II & 21 (14.9\%) & 30 (21.3\%) & \\
\hline IIla & $9(6.4 \%)$ & $20(14.2 \%)$ & \\
\hline IIIb & $3(2.1 \%)$ & $6(4.3 \%)$ & \\
\hline IVa & $4(2.8 \%)$ & $5(3.5 \%)$ & \\
\hline $\mathrm{IVb}$ & 0 & $2(1.4 \%)$ & \\
\hline V & $1(0.7 \%)$ & $2(1.4 \%)$ & \\
\hline Severe complication, III-V & 17 (12.1\%) & 35 (24.8\%) & 0.026 \\
\hline Postoperative hemorrhage & $4(2.8 \%)$ & $3(2.1 \%)$ & 1.000 \\
\hline Pancreatic fistula & & & 0.651 \\
\hline A & 24 (17.0\%) & $28(19.9 \%)$ & \\
\hline B & 15 (10.6\%) & $14(9.9 \%)$ & \\
\hline $\mathrm{C}$ & 0 & 0 & \\
\hline Wound infection & $1(0.7 \%)$ & $5(3.5 \%)$ & 0.214 \\
\hline Wound disruption & $1(0.7 \%)$ & $5(3.5 \%)$ & 0.214 \\
\hline Intra-abdominal infection & $8(5.7 \%)$ & $14(9.9 \%)$ & 0.266 \\
\hline Postoperative drainage & $11(7.8 \%)$ & $20(14.2 \%)$ & 0.090 \\
\hline Length of stay, $d$ & $4(4-6)$ & $7(5-9)$ & 0.026 \\
\hline Reoperation & $3(2.1 \%)$ & 7 (5.0\%) & 0.217 \\
\hline
\end{tabular}

Abbreviations: MIS, minimally invasive surgery.

The short-term benefits of MIDP vs ODP have been a topic of much interest. One systemic review and meta-analysis that included a total of 907 patients from eleven studies demonstrated comparable postoperative morbidity and mortality, as patients had the same incidence of pancreatic fistula, tumor recurrence, and postoperative mortality. ${ }^{3}$ MIDP was associated, however, with a shorter hospital stay and less blood loss. In contrast, a more recent meta-analysis by Drymousis and colleagues reported that patients who underwent laparoscopic surgery not only had lower blood loss and a shorter hospital stay but also lower overall morbidity. ${ }^{16}$ Both of these previous meta-analyses suffered, however, from the inclusion of mostly small single-center reports that failed to include statistical methodology, such as PSM to account for the heterogeneity among patients undergoing different surgical procedures. In contrast, the current study utilized PSM to help balance the MIDP and ODP cohorts. Of note, even after PSM, MIDP remained associated with decreased intraoperative blood loss, lower incidence of postoperative morbidity, and a shorter hospital stay compared with ODP. In addition, patients who underwent MIDP had a lower incidence of severe complications. While a previous report reported comparable postoperative morbidity and mortality between MIDP and ODP, the 
TABLE 3 Factors associated with tumor recurrence after curative resection of pancreatic neuroendocrine tumors (pNETs)

\begin{tabular}{|c|c|c|c|c|}
\hline & \multicolumn{2}{|c|}{ Univariate analysis } & \multicolumn{2}{|c|}{ Multivariable analysis } \\
\hline & HR $(95 \% \mathrm{Cl})$ & $P$ value & $\begin{array}{l}\text { HR } \\
(95 \% \mathrm{Cl})\end{array}$ & $P$ value \\
\hline Functional status & & 0.737 & & \\
\hline Nonfunctional & Ref. & & & \\
\hline Functional & $0.7(0.1-5.1)$ & & & \\
\hline Genetic syndrome & & 0.017 & & 0.034 \\
\hline Not associated & Ref. & & Ref & \\
\hline Associated & $1.9(1.1-3.3)$ & & $2.5(1.1-5.8)$ & \\
\hline Symptomatic & & 0.016 & & 0.701 \\
\hline No & Ref. & & Ref. & \\
\hline Yes & $1.8(1.1-2.8)$ & & $1.1(0.6-2.3)$ & \\
\hline Surgery technique & & $<0.001$ & & 0.033 \\
\hline Open & Ref. & & Ref. & \\
\hline $\begin{array}{l}\text { Laparoscopic/ } \\
\text { robotic }\end{array}$ & $0.2(0.1-0.5)$ & & $0.3(0.1-0.9)$ & \\
\hline $\begin{array}{l}\text { Major vascular } \\
\text { resection }\end{array}$ & $\begin{array}{l}5.0 \\
(2.0-12.5)\end{array}$ & 0.001 & $2.0(0.5-7.3)$ & 0.321 \\
\hline Splenectomy & $2.2(1.1-4.8)$ & 0.046 & $1.5(0.4-5.6)$ & 0.527 \\
\hline Tumor size, $\mathrm{cm}$ & & $<0.001$ & & 0.005 \\
\hline$\leq 3$ & Ref. & & Ref. & \\
\hline$>3$ & $5.6(3.4-9.3)$ & & $3.3(1.4-7.4)$ & \\
\hline Multiple lesions & $1.0(0.5-1.9)$ & 0.966 & & \\
\hline Surgical margin & & 0.009 & & 0.389 \\
\hline RO & Ref. & & Ref. & \\
\hline $\mathrm{R} 1$ & $2.0(1.2-3.4)$ & & $1.5(0.6-3.5)$ & \\
\hline \multicolumn{5}{|l|}{$\begin{array}{l}\text { Tumor } \\
\text { differentiation }\end{array}$} \\
\hline $\begin{array}{l}\text { Well } \\
\text { differentiated }\end{array}$ & Ref. & & Ref. & \\
\hline $\begin{array}{c}\text { Moderately } \\
\text { differentiated }\end{array}$ & $2.0(1.0-3.8)$ & 0.043 & $0.8(0.3-2.3)$ & 0.655 \\
\hline $\begin{array}{l}\text { Poorly } \\
\text { differentiated }\end{array}$ & $\begin{array}{l}8.1 \\
(2.9-22.6)\end{array}$ & $<0.001$ & $\begin{array}{l}3.8 \\
(0.4-33.4)\end{array}$ & 0.228 \\
\hline Nodal status & & $<0.001$ & & 0.517 \\
\hline NO & Ref. & & Ref. & \\
\hline N1 & $2.8(1.7-4.4)$ & & $0.8(0.3-1.7)$ & \\
\hline \multicolumn{5}{|l|}{ WHO classification } \\
\hline G1 & Ref. & & Ref. & \\
\hline $\mathrm{G} 2$ & $3.2(1.8-5.6)$ & $<0.001$ & $2.1(0.9-4.8)$ & 0.092 \\
\hline G3 & $\begin{array}{l}12.8 \\
(5.3-30.5)\end{array}$ & $<0.001$ & $\begin{array}{l}4.1 \\
(0.8-20.7)\end{array}$ & 0.088 \\
\hline $\begin{array}{l}\text { Lymph-vascular } \\
\text { invasion }\end{array}$ & $3.9(2.3-6.7)$ & $<0.001$ & $1.5(0.7-3.5)$ & 0.308 \\
\hline Perineural invasion & $1.6(0.9-3.1)$ & 0.139 & & \\
\hline
\end{tabular}

study had suggested a superiority of MIDP over ODP for pancreatic ductal adenocarcinoma in terms of intraoperative blood loss and hospital stay. ${ }^{23} \mathrm{~A}$ separate study by Xoufras et $\mathrm{al}^{24}$ that examined patients who underwent DP for pNET noted that laparoscopic DP was associated with a lower incidence of postoperative complications and a shorter hospital stay vs ODP. Collectively the data strongly suggest that MIDP may be superior to ODP with regard to perioperative outcomes including blood loss, complications, and length-of-stay.

The current study showed a widespread and increased utilization of MIDP for pNET among major centers in the United States. In addition to the overall increase in the utilization of MIDP, the conversion rate decreased roughly from $20.0 \%$ before 2008 to $7.8 \%$ in 2009-2012 and 7.6\% in 2013-2016. Braga et al $^{25}$ noted an overall conversion rate of $23.3 \%$, yet noted that the conversion rate dropped significantly after the first ten laparoscopic DP cases. In contrast, Shakir et $\mathrm{al}^{26}$ noted that robotic DP required 40 cases to optimize outcomes, such as operative time and blood loss. Interestingly, a temporal trend in the number of lymph nodes evaluated also increased over the time periods examined, whereas the incidence of severe complications and length-of-stay also both decreased. These data indicated that the increased use of MIDP over time has paralleled an increase in experience that has further augmented the beneficial perioperative effects of the minimally invasive approach.

While the favorable perioperative outcomes associated with MIDP have been generally accepted, the oncologic outcomes of MIDP vs ODP for pNET remain undetermined. Most previous reports reported only small groups of patients and data on surgical factors (eg margin status, lymphadenectomy) were not well defined. The data from our multi-institutional series demonstrated that some oncologic and surgical factors were comparable among patients who underwent MIDP vs ODP, yet other factors varied. For example, patients who underwent MIDP had a higher rate of RO resection, which was likely because of patients with ODP presenting with more advanced disease (Table 1). The data did suggest, however, that MIDP was at least as effective as ODP in obtaining an adequate margin and lymph node evaluation. Patients who underwent ODP had a higher risk of recurrence that was almost undoubtedly related to differences in underlying tumor factors. To minimize the patient selection bias, we utilized PSM to create more balanced cohorts for comparison. On PSM, after balancing many tumor and surgical factors, patients who underwent MIDP still had a lower incidence of tumor recurrence, yet OS was equivalent to patients who underwent ODP. In a separate study by Xourafas et al, ${ }^{24}$ patients undergoing MIDP $(n=78)$ for pNET similarly had comparable recurrence and OS vs individuals who underwent ODP. Interestingly, the incidence of recurrence after MIDP was similar in the current study compared with the data from Xourafas et $\mathrm{al}^{24}$ (3.7\% vs $4 \%$ ), yet recurrence among the ODP group was considerably higher in the current study ( $19.1 \%$ vs $4 \%)$. The difference in recurrence was undoubtedly multifactorial and was 
likely related to differences in patient selection. For example, the incidence of R1 resection among patients undergoing ODP was higher in the current study than the study by Xourafas et $\mathrm{al}^{24}(16.6 \%$ vs $7 \%$ ). Of note, similar to the current study, DiNorcia et $a^{27}$ reported a higher tumor recurrence rate (15.3\%) after open surgery vs minimally invasive procedures (4.4\%) for pNET. These authors postulated that the higher recurrence rate in the open surgery group was explained by more advanced disease on presentation. ${ }^{27}$ The data from the current study, as well as several previous reports, strongly suggest at least non-inferior oncologic outcomes for MIDP vs ODP for the resection of pNET.

The current study had several limitations. Although the multiinstitutional collaboration increased the sample size and generalizability of the results, possible inconsistency in patient selection, choice of MIDP or ODP, surgical skills and procedures, pathologic assessment and reporting, as well as postoperative surveillance likely existed. While known tumor and surgery-related factors were matched between the MIDP and ODP groups using PSM, patients undergoing ODP still had pNET with more advanced tumor features vs MIDP in the matched cohort. Patients with smaller and low-grade tumor were more likely to undergo MIS. As such, residual confounding-by-indication persisted in comparing the MIDP vs ODP groups. Moreover, the outcome of patients undergoing MIS is largely influenced by technical skills. However, the number of surgeons in each institution and their learning curves were not available in the current database. The current study also focused on DP; future studies will, therefore, need to assess the short- and long-term outcomes of minimally invasive vs open procedures for more complex surgery, such as PD for pNET.

In conclusion, the utilization of MIDP increased to more than one-half of all surgical procedures for pNET over the last two decades. Current conversion rates were less than $10 \%$ and MIDP was associated with less blood loss, a lower incidence of postoperative morbidity, and a shorter hospital stay compared with ODP. The data from the current study demonstrated comparable oncologic surgical metrics, as well as similar long-term OS among patients undergoing MIDP over ODP in the treatment of pNET. As such, a minimally invasive approach to pNET tumors should be considered as the surgical approach of choice when technically feasible.

\section{ACKNOWLEDGMENT}

Xu-Feng Zhang was supported by the Clinical Research Award of the First Affiliated Hospital of Xi'an Jiaotong University of China (No. XJTU1AF-CRF-2017-004).

\section{CONFLICT OF INTERESTS}

The authors declare that there are no conflict of interests.

\section{AUTHOR CONTRIBUTIONS}

$\mathrm{X}-\mathrm{FZ}$, YL, and TMP conceptualized, designed, and carried out the acquisition, analysis, and interpretation of the data. AGL-A, GP, EM, $\mathrm{FR}, \mathrm{ZK}, \mathrm{SW}, \mathrm{AF}, \mathrm{RF}, \mathrm{BAK}, \mathrm{KI}, \mathrm{PMS}, \mathrm{CC}$, and MB wrote the manuscript, carried out acquisition and interpretation of the data. CRS, MD, SKM, and TM conceptualized, designed, analyzed, and interpreted the data, and revised the article.

\section{DATA AVAILABILITY STATEMENT}

The data that support the findings of this study are available on request from the corresponding author. The data are not publicly available because of privacy or ethical restrictions.

\section{ORCID}

Xu-Feng Zhang (D) http://orcid.org/0000-0002-4483-7326

\section{REFERENCES}

1. Milan SA, Yeo CJ. Neuroendocrine tumors of the pancreas. Curr Opin Oncol. 2012;24:46-55.

2. Yao JC, Eisner MP, Leary $C$, et al. Population-based study of islet cell carcinoma. Ann Surg Oncol. 2007;14:3492-3500.

3. Tamburrino D, Partelli S, Renzi C, et al. Systematic review and metaanalysis on laparoscopic pancreatic resections for neuroendocrine neoplasms (PNENs). Expert Rev Gastroenterol Hepatol. 2017;11:65-73.

4. Dasari A, Shen C, Halperin D, et al. Trends in the incidence, prevalence, and survival outcomes in patients with neuroendocrine tumors in the United States. JAMA Oncol. 2017;3:1335-1342.

5. Tsikitis VL, Wertheim BC, Guerrero MA. Trends of incidence and survival of gastrointestinal neuroendocrine tumors in the United States: a seer analysis. J Cancer. 2012;3:292-302.

6. Hill JS, McPhee JT, McDade TP, et al. Pancreatic neuroendocrine tumors: the impact of surgical resection on survival. Cancer. 2009;115:741-751.

7. Schurr PG, Strate T, Rese K, et al. Aggressive surgery improves longterm survival in neuroendocrine pancreatic tumors: an institutional experience. Ann Surg. 2007;245:273-281.

8. Bagante F, Spolverato G, Merath K, et al. Neuroendocrine liver metastasis: The chance to be cured after liver surgery. J Surg Oncol. 2017;115:687-695.

9. Xiang JX, Zhang XF, Beal EW, et al. Hepatic resection for nonfunctional neuroendocrine liver metastasis: does the presence of unresected primary tumor or extrahepatic metastatic disease matter? Ann Surg Oncol. 2018;25:3928-3935.

10. Szeliga J, Jackowski M. Pancreatic neuroendocrine neoplasms: a role of laparoscopy in surgical treatment: review. Surg Laparosc Endosc Percutan Tech. 2018;28:147-152.

11. Haugvik SP, Marangos IP, Rosok BI, et al. Long-term outcome of laparoscopic surgery for pancreatic neuroendocrine tumors. World J Surg. 2013;37:582-590.

12. Cuschieri A. Laparoscopic surgery of the pancreas. J R Coll Surg Edinb. 1994;39:178-184.

13. Soreide K, Olsen F, Nymo LS, et al. A nationwide cohort study of resection rates and short-term outcomes in open and laparoscopic distal pancreatectomy. HPB (Oxford). 2018. https://doi.org/10.1016/j. hpb.2018.10.006 
14. Klompmaker S, van Hilst J, Wellner UF, et al. Outcomes after minimally-invasive versus open pancreatoduodenectomy: a paneuropean propensity score matched study. Ann Surg. 2018:1. https://doi.org/10.1097/SLA.000000000000285

15. Cienfuegos JA, Salguero J, Nunez-Cordoba JM, et al. Short- and longterm outcomes of laparoscopic organ-sparing resection in pancreatic neuroendocrine tumors: a single-center experience. Surg Endosc. 2017;31:3847-3857.

16. Drymousis $P$, Raptis DA, Spalding D, et al. Laparoscopic versus open pancreas resection for pancreatic neuroendocrine tumours: a systematic review and meta-analysis. HPB (Oxford). 2014;16: 397-406.

17. Zhang XF, Wu Z, Cloyd J, et al. Margin status and long-term prognosis of primary pancreatic neuroendocrine tumor after curative resection: results from the US Neuroendocrine Tumor Study Group. Surgery. 2019;165:548-556.

18. The Royal College of Pathologists. Standards and Minimum Datasets for Reporting Cancers. Minimum Dataset for the Histopathological Reporting of Pancreatic, Ampulla of Vater and Bile Duct Carcinoma. London: London, United Kingdom: The Royal College of Pathologists, 2002.

19. Kloppel G. Classification and pathology of gastroenteropancreatic neuroendocrine neoplasms. Endocr Relat Cancer. 2011;18(Suppl 1): S1-16.

20. Dindo D, Demartines N, Clavien PA. Classification of surgical complications: a new proposal with evaluation in a cohort of 6336 patients and results of a survey. Ann Surg. 2004;240: 205-213.

21. Bassi C, Dervenis C, Butturini G, et al. Postoperative pancreatic fistula: an international study group (ISGPF) definition. Surgery. 2005;138:8-13.

22. Wente MN, Veit JA, Bassi C, et al. Postpancreatectomy hemorrhage (PPH): an International Study Group of Pancreatic Surgery (ISGPS) definition. Surgery. 2007;142:20-25.
23. van Hilst J, Korrel M, de Rooij T, et al. Oncologic outcomes of minimally invasive versus open distal pancreatectomy for pancreatic ductal adenocarcinoma: a systematic review and meta-analysis. Eur $J$ Surg Oncol. 2018;45(5):719-727.

24. Xourafas D, Tavakkoli A, Clancy TE, Ashley SW. Distal pancreatic resection for neuroendocrine tumors: is laparoscopic really better than open? J Gastrointest Surg. 2015;19:831-840.

25. Braga M, Ridolfi C, Balzano G, et al. Learning curve for laparoscopic distal pancreatectomy in a high-volume hospital. Updates Surg. 2012;64:179-183.

26. Shakir M, Boone BA, Polanco PM, et al. The learning curve for robotic distal pancreatectomy: an analysis of outcomes of the first 100 consecutive cases at a high-volume pancreatic centre. HPB (Oxford). 2015;17:580-586.

27. DiNorcia J, Lee MK, Reavey PL, et al. One hundred thirty resections for pancreatic neuroendocrine tumor: evaluating the impact of minimally invasive and parenchyma-sparing techniques. J Gastrointest Surg. 2010;14:1536-1546.

\section{SUPPORTING INFORMATION}

Additional supporting information may be found online in the Supporting Information section at the end of the article.

How to cite this article: Zhang X-F, Lopez-Aguiar AG,

Poultsides $\mathrm{G}$, et al. Minimally invasive versus open distal pancreatectomy for pancreatic neuroendocrine tumors: An analysis from the U.S. neuroendocrine tumor study group. J Surg Oncol. 2019;120:231-240. https://doi.org/10.1002/jso.25481 